\title{
Semi-expendable Unmanned Aerial Vehicle for forest fire suppression
}

\author{
D. Benavente \\ Founder, Embention Sistemas Inteligentes, S.L., Spain
}

\begin{abstract}
Rapid detection systems and other surveillance and real-time monitoring systems have become more and more a reality in recent years. The information generated is very useful for avoiding risks and to optimally manage the available firefighting resources. However, there are lots of scenarios for which a rapid and effective response is not possible because of strong winds, the absence of daylight or difficulty in gaining access to a specific location with conventional aerial assets. This paper presents a new forest fire suppression tool capable of handling all these factors: Flamingo (Forest fLAMes INtelliGent Outputter), a low-cost semi-expendable Unmanned Aerial Vehicle (UAV) with a single-use deposit loaded with fire retardants.
\end{abstract}

Keywords: Flamingo, forest fire suppression technology, civil UAV, guided bomb, severe winds, night operations, Veronte, autopilot.

\section{Introduction}

In recent years technology has been entering the forest fire world with rapid detection systems, real-time monitoring systems, etc. These systems can indeed contribute to the avoidance of risks and elaborate optimal suppression strategies thanks to the information they provide. What we are presenting here is technology applied to the suppression area.

Our approach tries to fill the gap left by conventional aerial means whose operation is too dangerous in the presence of strong winds and/or during nighttime. This gap has been also targeted by other systems like PCADS [4]. Night operation in particular is one of the key points identified for the near future by many forest fire aerial contractors. 
Concerning our approach, the basics of the technology have been used for many years in the past for military purposes. In fact, the way Flamingo works is quite similar to that of any guided bomb being used nowadays. However, we prefer to say that Flamingo is a semi-expendable UAV that mainly consists of 2 parts: a single-use deposit and a re-usable control unit including the fins, servos and autopilot responsible for getting the load of fire retardants right to the coordinates established a couple of seconds before flight.

Although the feasibility of precise self guidance towards a target has been proven with guided bombs unfortunately many times, the cost of this technology might result too high for wildfire suppression. Keeping the overall unit cost well under 1KEUR while keeping flight performances posed a big design challenge on many areas because no fancy materials or precision sensors could be used onboard.

\section{System use}

In its current version, each Flamingo unit contains 206 litres of fire retardant. Although Flamingo guides itself by deflecting its fins in the air, it is not provided with propulsion means onboard. This means that in order for Flamingo to fly, it needs to be dropped from an aircraft from an altitude of approximately $10000 \mathrm{ft}$. The aircraft we are considering for operation (e.g. CN-235, C-295, C-130 Hercules, A400M, etc) could carry from 20 up to 100 Flamingo units resulting in more than 20000 litres of fire retardant delivered per flight.

The baseline use is as follows: once a forest fire is detected and the system is requested for operation, a cargo aircraft is loaded with a set of Flamingo units. All these units exchange data within a low-power wireless local network. Apart from the set of Flamingo units, the aircraft and its cargo-like launch system onboard, there is still a key part of the system to be mentioned: the Flamingo Air Control Station (FACS) and the Flamingo Ground Control Station (FGCS).

The Flamingo Air Control Station is responsible for transmitting independent target coordinates to each of the Flamingo units onboard. Therefore, FACS also constitutes a node in the wireless local network onboard. In addition, FACS holds a long-range communications link to the FGCS. All the fire information available is displayed to the system operators at both the FACS and FGCS using a GIS-based software tool. In addition, the software allows the user to define a direct attack or an indirect suppression strategy which will result in a set of coordinates to be given individually to each of the Flamingo units inside the aircraft just before launch.

As the aircraft flies towards the fire (at $10000 \mathrm{ft}$ altitude), a launch window gets open. At that moment, the set of target coordinates are updated with the latest information available and all the Flamingo units are dropped like cargo. After a couple of seconds after launch the transient gets cancelled and each Flamingo starts to move its fins to get to its target. In order to achieve this, Flamingo is equipped with our autopilot Veronte, which runs the guidance, navigation and control algorithms needed in real time. The navigation algorithm computes at every moment the position, velocity and attitude of Flamingo by 
making a fusion of GPS, magnetic, pressure and inertial measurements. The guidance algorithm basically ensures that Flamingo can always reach the target at any given moment. Lastly the control algorithm regulates the difference between the estimated variables and the desired ones.

In less than a minute Flamingo hits ground at more than $300 \mathrm{~km} / \mathrm{h}$ breaking the deposit and helping spread the retardant. A couple of seconds before the impact, the control unit gets separated from the deposit right before it deploys a parachute in order to absorb energy itself and avoid a strong impact.

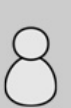

operator

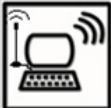

FACS
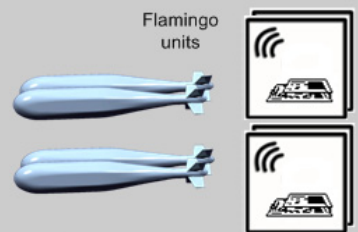

Flight Segment

Ground Segment

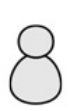

operator

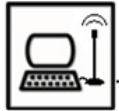

FGCS
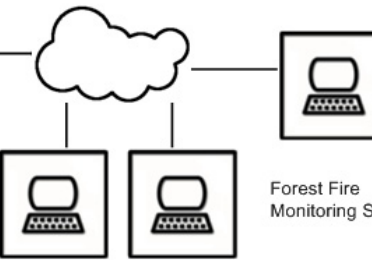

Forest Fire

Monitoring Systems

Figure 1: $\quad$ Flamingo UAV architecture.
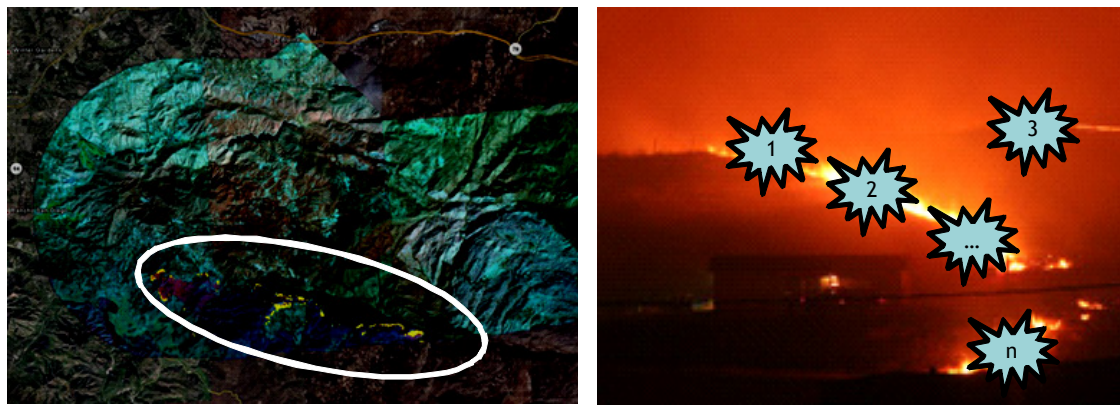

Figure 2: $\quad$ System use: strategy and allocation. 


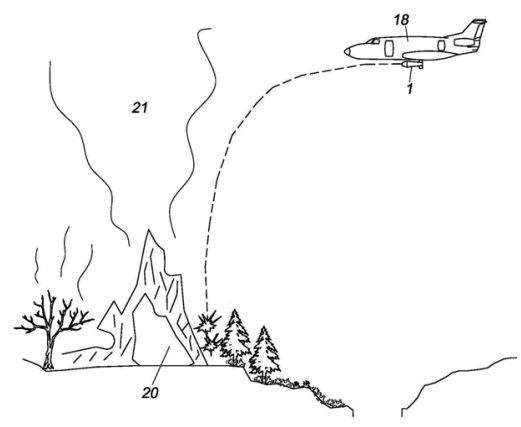

Figure 3: Direct suppression strategy.

\section{About Flamingo}

The main element of the system presented is Flamingo. As we mentioned earlier we like to say that Flamingo is a semi-expendable UAV composed of 2 main parts: the Suppression Unit and the Control Unit.

\subsection{Suppression unit}

It is a slender deposit containing the UAV payload, that is, the retardant. Since it is a single-use component it has been designed to minimize its unit cost while its structure is able to withstand a $4.5 \mathrm{~g}$ manoeuvre while in the aircraft [1]. This component is also the main responsible for inherent aerodynamic stability and controllability. Furthermore, it has to ensure retardant tightness at every time throughout the mission. Minimizing the cost subject to all these constraints was not an easy problem to solve. As of today, the solution we have reached consists of a single piece produced with a rotomoulding technique.

\subsection{Control unit}

It is the piece adding the intelligence and control capabilities to Flamingo. It contains a set of aerodynamic surfaces deflected by a set of servos via a transmission system. These fins are moved by our Veronte autopilot as an outcome of our embedded algorithms which take into account not only the target coordinates but also the measurements from its onboard mounted sensors: GPS, inertial measurement unit, magnetometer, and air data system. Veronte is also responsible for separating the control unit from the deposit at the right time and deploying a parachute right away to avoid a strong impact. It is important to mention that the control unit by itself is also a structural piece because it has to transmit all aerodynamic loads from the fins to the deposit. 

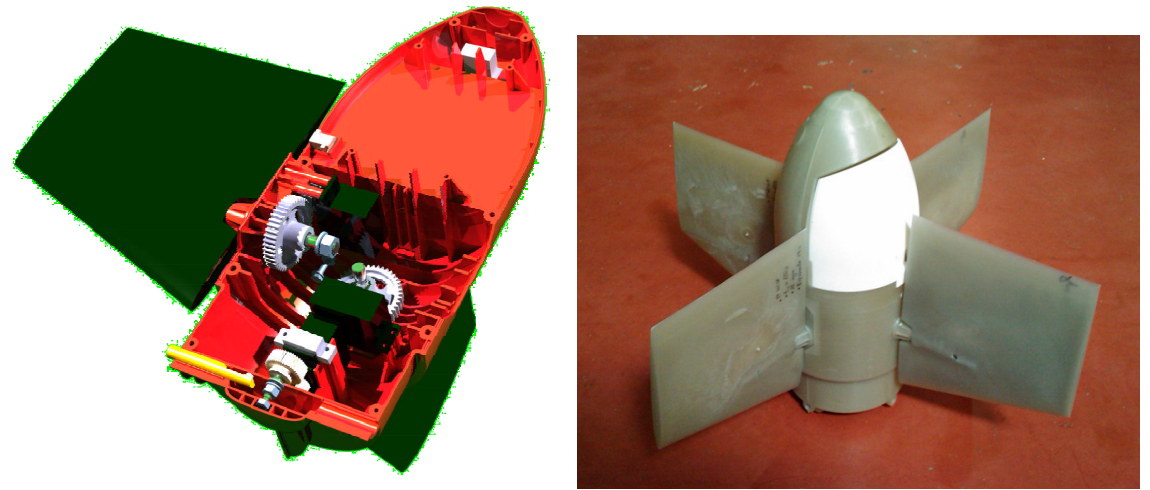

Figure 4: $\quad$ Suppression unit and control unit.
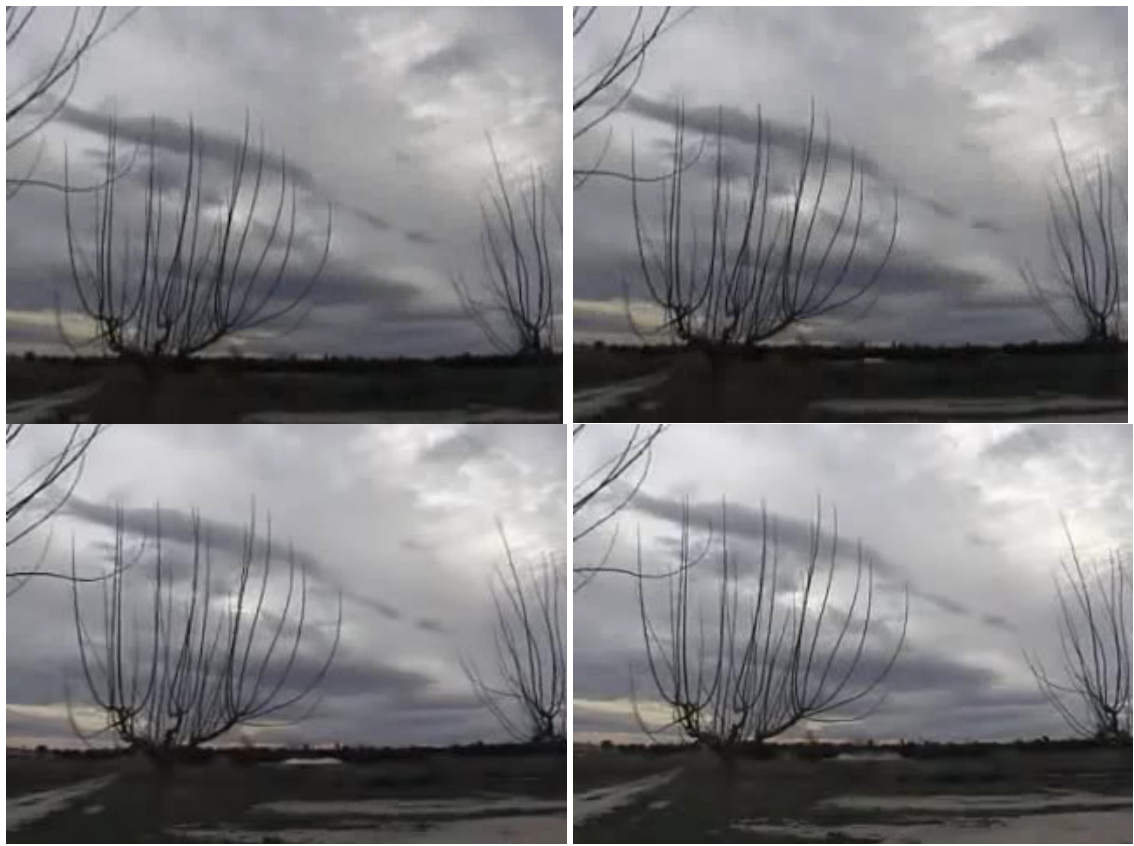

Figure 5: Frames from 5-liter prototype impact on 20m x 20m concrete platform. 


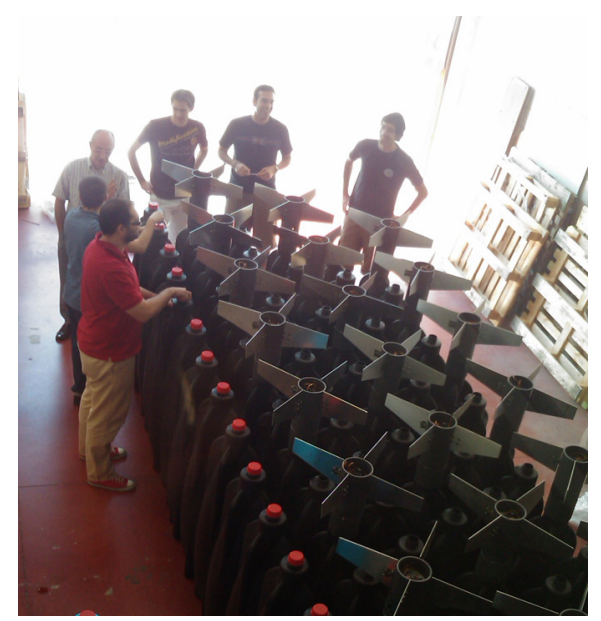

Figure 6: Beta series ready to fly.

\section{Current status}

After a conceptual 5-liter prototype and a beta series of 60 litres, we developed our product scaled on a 206-liter deposit. Both the suppression and control units were carefully designed taking into account the lessons learnt with the prototypes and the beta series.

At this moment the product Flamingo is involved in a qualification process for aeronautic products at INTA (Instituto Nacional de Técnica Aeroespacial, Spain) for its integration and operation with the EADS CASA C-212 aircraft using a cargo door drop system [2].

As part of the qualification process, full system tests will start shortly with the collaboration of INTA, where the extinguishing power of each deposit will constitute one of the main results. One of the main difficulties we have faced so far is the complexity to carry out tests. Not only it is not easy to obtain burn permits in Spain but also it is extremely difficult to get approvals for experimental bombs to be dropped from an airplane. However, since it was one of the main challenges in our project we managed to test our autopilot and control system in flight with our 5-litre prototypes from $1800 \mathrm{ft}$.

\section{References}

[1] A. Bedmar, D. Benavente, M. Breen, M. García, J. González, D. Gutiérrez, Flamingo Technical Specification, david.benavente@embention.com

[2] L. Dávila, R. Dorado, B. Marqués, AER/PRO/7130/003/INTA

[3] RD 2218/2004, Reglamento de Aeronavegabilidad de la Defensa (RAD)

[4] PCADS, http://www.caylym.com/

[5] MIL-HDBK-1791 (USAF) Designing for Internal Aerial Delivery in FixedWing Aircraft 